\title{
Validity of self-reported and objectively measured sedentary behavior in pregnancy
}

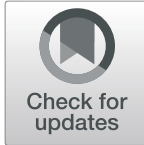

Bethany Barone Gibbs ${ }^{1,2^{*}}$ (D, Joshua L. Paley ${ }^{1}$, Melissa A. Jones ${ }^{1}$, Kara M. Whitaker ${ }^{3}$, Christopher P. Connolly ${ }^{4}$ and Janet M. Catov ${ }^{5}$

\begin{abstract}
Background: Sedentary behavior (SED) is a potential risk factor for poor pregnancy outcomes. We evaluated the validity of several common and one new method to assess SED across three trimesters of pregnancy.

Methods: This cohort study of pregnant women measured objective and self-reported SED each trimester via thigh-worn activPAL3 micro (criterion), waist-worn Actigraph GT3X, and self-report from the Pregnancy Physical Activity Questionnaire (PPAQ) and the de novo Sedentary Behavior Two Domain Questionnaire (SB2D). SED (hours per day) and percent time in SED (SED\%) from activPAL were compared to GT3X, SB2D, and PPAQ using Pearson's $r$, ICC, Bland-Altman analysis, and comparison of criterion SED and SED\% across tertiles of alternative methods.

Results: Fifty-eight women (mean age $31.5 \pm 4.8$ years; pre-pregnancy BMI $25.1 \pm 5.6 \mathrm{~kg} / \mathrm{m}^{2} ; 76 \%$ white) provided three trimesters of valid activPAL data. Compared to activPAL, GT3X had agreement ranging from $r=0.54-0.66$ and ICC $=0.52-0.65$. Bland-Altman plots revealed small mean differences and unpatterned errors, but wide limits of agreement (greater than $\pm 2 \mathrm{~h}$ and $\pm 15 \%$ ). The SB2D and PPAQ had $r<0.5$ and ICC $<0.3$ vs. activPAL SED, with lower agreement during the 2nd and 3rd trimesters, and performed poorly in Bland-Altman analyses. SED\% from the modified SB2D performed best of the self-reported instruments with modest mean differences, $r$ ranging from 0.55 to 0.60 , and ICCS from 0.31-0.33; though, limits of agreement were greater than $\pm 35 \%$. Significant trends in activPAL SED were observed across increasing tertiles of SB2D SED in the 1st and 3rd trimesters (both $p \leq 0.001$ ), but not the 2nd trimester ( $p=0.425$ ); and for PPAQ SED in the 1st and 2nd trimesters (both $p<0.05$ ), but not the 3rd trimester $(p=0.158)$. AcitvPAL SED and SED\% increased significantly across tertiles of GT3X SED and SED\% as well as SB2D SED\% (all $p$-for-trend $\leq 0.001$ ).
\end{abstract}

Conclusions: Compared to activPAL, waist-worn GT3X produced moderate agreement, though similar mean estimates of SED across pregnancy. Self-report questionnaires had large absolute error and wide limits of agreement for SED hr./day; SB2D measurement of SED\% was the best self-report method. These data suggest activPAL be used to measure SED when possible, followed by GT3X, and - when necessary - SB2D assessing SED\% in pregnancy.

Trial registration: www.clinicaltrials.gov NCT03084302 on 3/20/2017.

Keywords: activPAL, Accelerometer, Sedentary time, Trimester, Self-report questionnaire

\footnotetext{
* Correspondence: bbarone@pitt.edu

'Department of Health and Physical Activity, Clinical and Translational

Sciences, University of Pittsburgh, 32 Oak Hill Court, Room 220, Pittsburgh,

PA 15261, USA

${ }^{2}$ Department of Clinical and Translational Sciences, University of Pittsburgh,

Pittsburgh, USA

Full list of author information is available at the end of the article
}

(c) The Author(s). 2020 Open Access This article is distributed under the terms of the Creative Commons Attribution 4.0 International License (http://creativecommons.org/licenses/by/4.0/), which permits unrestricted use, distribution, and reproduction in any medium, provided you give appropriate credit to the original author(s) and the source, provide a link to the Creative Commons license, and indicate if changes were made. The Creative Commons Public Domain Dedication waiver (http://creativecommons.org/publicdomain/zero/1.0/) applies to the data made available in this article, unless otherwise stated. 


\section{Introduction}

Sedentary behavior, defined as waking behavior in a seated, lying, or reclining posture and at low intensity (< 1.5 metabolic equivalents [METs]) [1], is an emerging risk factor for outcomes such as weight gain [2], cardiometabolic disease [3, 4], and depression [5]. Pregnancy is a biologically relevant period during which adverse outcomes, such as excessive gestational weight gain, gestational hypertension, and gestational diabetes, can manifest [6]. Though regular physical activity during pregnancy can protect against these outcomes [7], most pregnant women do not achieve recommended levels [8, 9]. Preliminary data suggests that pregnant women also engage in high levels of sedentary behavior $[8,10]$, independent of engagement in moderate-to-vigorous intensity physical activity. Thus, sedentary behavior reduction may be a distinct and potentially feasible behavioral target to improve pregnancy health.

Despite the possibility that sedentary behavior is a novel risk factor during pregnancy, there is a dearth of high quality research evaluating sedentary behavior patterns across gestation and associated outcomes in pregnant women. A recent systematic review concluded that, though more than 25 studies have evaluated sedentary behavior in pregnancy, substantial heterogeneity in methodology limits the ability to synthesize findings [11]. The primary recommendation of this review was that studies using 'robust methodology for quantifying sedentary behavior' are most needed [11]. This highlights an overall challenge in sedentary behavior research where definitions and best practice assessment methodology have only recently emerged $[1,12]$.

Existing studies of sedentary behavior in pregnant women have used both self-report questionnaires and objective monitors. These have commonly included selfreport by the sedentary behavior subscale on the Pregnancy Physical Activity Questionnaire (PPAQ) [13, 14] or an accelerometer [10, 15-17]. However, these methodologies have distinct limitations as compared to the current best practice of a thigh-mounted inclinometer and accelerometer (e.g., activPAL), which has been used in fewer pregnancy studies $[11,18]$. The activPAL is preferred due to its capability of capturing both the postural (seated/reclining/lying) and intensity (<1.5 METs) aspects of the current consensus definition of sedentary behavior [1]. Daily participation in sedentary behavior is known to be poorly estimated by self-report instruments in non-pregnant populations [19], a phenomenon that may reflect its high frequency and intermittency throughout the day. While objective monitoring is thus preferred to quantify total sedentary time [20], the commonly used method of waist- or wrist-worn accelerometry (e.g., Actigraph) is limited in that it only measures the absence of movement (i.e., stationary behavior) rather than the definition of sedentary behavior that includes posture and intensity [1]. This may be further compromised in the later stages of pregnancy where the standard position of waist-worn accelerometry must be adjusted [21].

Unfortunately, among pregnant women, the ability of self-reported or accelerometer-measured sedentary behavior as compared to activPAL to estimate daily duration or rank women by level of sedentary behavior is currently unknown. This is important for both interpretation of available data and also for planning of future research relating sedentary behavior to maternal-fetal outcomes. To address this gap, we used data from an ongoing cohort study measuring objective sedentary behavior in pregnant women across three trimesters. We aimed to evaluate agreement between accelerometer-measured sedentary behavior as well as several self-report instruments as compared to best practice assessment with a thigh-mounted activPAL monitor in each trimester of pregnancy.

\section{Methods \\ Participants and setting}

This study uses data from a subsample of participants enrolled in the Monitoring Movement and Health Study (MoM Health), a longitudinal cohort study characterizing sedentary behavior, physical activity, and pregnancy health outcomes across each trimester of pregnancy (clinicaltrials.gov identifier: NCT03084302). Pregnant women were recruited for the parent study using fliers at and around obstetrics and gynecology practices, word-of-mouth, a University-based research registry, and emails to University of Pittsburgh employees. Women were eligible to participate in the MoM Health study if they were: less than 14 weeks pregnant, planning to have prenatal care and deliver at a University of Pittsburgh Medical Center facility, and able to attend all study visits. Women were excluded if they had severely limited mobility (defined as unable to walk $1 / 2$ mile or up 2 flights of steps), were currently taking medication to control blood pressure or diabetes, had a severe medical condition (e.g., chronic obstructive pulmonary disease, renal disease), or if they were currently participating in another research study intending to modify their lifestyle behavior. All procedures were approved by the University of Pittsburgh Institutional Review Board and all participants provided written informed consent prior to participating in the study.

MoM Health Study participants attended three study visits: first trimester (between 8 and 13 weeks); second trimester (between 20 and 22 weeks); and third trimester (between 32 and 34 weeks). To be included in the current validation study, participants were required to have completed all three study visits with valid criterion 
sedentary behavior measurement by the activPAL3 micro (described below). Of the first 65 enrolled participants that completed study visits during all three trimesters, 58 women met this criteria. Seven women were excluded due to device failure $(n=5)$ or lost monitors $(n=2)$ at one of the three visits.

\section{Measures}

\section{Demographics and clinical measures}

Participant characteristics were self-reported on standard questionnaires. Pre-pregnancy weight was abstracted from participant medical records and height measured by stadiometer with shoes removed at the first trimester assessment visit. These were used to calculate prepregnancy body mass index (BMI) as $\mathrm{kg} / \mathrm{m}^{2}$.

\section{activPAL3 micro (criterion)}

Measurement of sedentary behavior used the activPAL3 micro (PALtechnologies, Glastgow, Scotland) thighmounted accelerometer and inclinometer as well as published protocol recommendations [1, 12, 22]. During each visit, participants first received verbal and written instructions then self-applied the monitor to the anterior thigh using a provided Tegaderm ${ }^{\circ}$ dressing. The research personnel then confirmed correct placement. Participants were instructed to wear the monitor $24 \mathrm{~h}$ per day, for 7 complete days, with removal only for swimming. Seven additional Tegaderm ${ }^{\circ}$ dressings were provided and participants were instructed that they could change dressings and alternate legs as needed if the dressing came loose or the skin underneath became irritated. During monitor wear, participants completed a diary that reported time awoke in the morning, time went to sleep, naps, and any removal of the device. Event data from the activPAL were exported, cleaned, and reduced by trained research personnel using standardized procedures that combined diary and objective data to identify waking wear periods across the monitoring interval [12]. For each wear day, daily sedentary time (SED) in hours per day was calculated as the sum of all SED intervals during waking hours. A minimum of 4 days with at least $10 \mathrm{~h}$ per day of monitoring was required to be considered valid. Estimates of daily SED and wear time (hours per day) as well as percentage of time sedentary (SED\% = SED divided by wear time) were averaged over valid days.

\section{Actigraph GT3X}

Participants were instructed to wear the Actigraph GT3X accelerometer (Actigraph, Pensacola, FL) on an elastic belt fastened snugly to their torso, directly over the right side of their right kneecap during all waking hours, with removal while sleeping and during water activities (bathing or swimming). The GT3X was worn concurrently with the activPAL over 7 complete days. Because of changing anthropometry across pregnancy, and from previous research by the study team [23], pictures were provided to aid in correct device placement (with elastic belt below belly, as needed). Accelerometer data (60-s epochs) were exported and reduced using ActiLife Software v6.13.3. Nonwear time was identified using an automated protocol of any period with at least 60 consecutive minutes of 0 counts per minute (cpm), with an allowance for $2 \mathrm{~min}$ of $<100 \mathrm{cpm}$ [24]. SED was defined as any 60 -s epoch with $<100 \mathrm{cpm}$ during valid wear time $[24,25]$. A minimum of 4 days with at least $10 \mathrm{~h}$ per day of monitoring was required to be included in analysis [24]. Daily estimates of SED, wear time (hours per day), and \%SED (SED divided by wear time) were averaged over valid days. Of the 58 women meeting criteria for valid activPAL data at each assessment visit, 57,56 , and 51 women had valid GT3X data at the 1st, 2nd, and 3rd trimesters respectively. Data were missing due to insufficient wear time $(n=7)$ or device failures $(n=3)$.

\section{Self-report}

Sedentary behavior was assessed using two self-report methods. First, the sedentary behavior subscale from the Pregnancy Physical Activity Questionnaire (PPAQ) [26] was included as it is a validated and commonly used instrument to assess time spent in participating in a variety household/caregiving, occupational, sports/exercise, and transportation activities among pregnant women [27-29]. The PPAQ estimates SED in hours per day by summing duration $\mathrm{x}$ intensity for questions 12 , 13, 30, and 31 (if open-ended questions are $<1.5$ METs) [26]. Reflecting the 2017 consensus definition of sedentary behavior [1] published after the PPAQ sedentary behavior subscale was published in 2004, we additionally summed responses to PPAQ questions 11 (sitting using a computer or writing while not at work), 22 (driving or riding in a car or bus), and 32 (sitting at work or in class) as recommended by the DAPA Measurement Toolkit (https:/dapa-toolkit.mrc.ac.uk/pdf/pa/ PPAQ_instructions_1.pdf). Of note, we slightly modified the scoring algorithm which includes multiplying durations by intensity for each item on the sedentary behavior subscale. Rather, we chose to only sum durations as this was most comparable to the duration estimate from our criterion measure. When we repeated analyses using the published scoring algorithm and comparing PPAQ SED MET-hours per day to activPAL SED hours per day, results were either less or similarly correlated to the criterion measure (data not shown). One participant missed two SED questions on the PPAQ during the 2nd trimester and was not included 
for comparisons between activPAL and PPAQ for that visit $(n=57)$.

Second, at the beginning of the MoM Health Study, two short de novo instruments were developed to assess SED with the purpose of validation among pregnant women (hereafter referred to as the Sedentary Behavior Two Domain Questionnaire, SB2D). We used the language from the sedentary behavior question from the Global Physical Activity Questionnaire (GPAQ), [30] which is commonly used to assess sedentary behavior [31,32] and which we found to be superior to a multi-domain sedentary behavior questionnaire in a previous validation study among young adults with a similar mean age to our population [33]. Next, we modified the question to capture SED (hours per day) separately on work (if applicable) and non-work days, similar to another sedentary behavior questionnaire by Whitfield, et al., [34] as employment is an important determinant of sedentary behavior in adult populations [35]. Last, in agreement with a recent systematic review of sedentary behavior questionnaire taxonomy that concluded SED\% rather than absolute SED is recommended for population surveillance [36], we repeated the two-item instrument using Likert-type answers taken from the Canadian Fitness Survey [37] (response options were: almost none of the time, $1 / 4$ of the time, $1 / 2$ of the time, $3 / 4$ of the time, almost all of the time). To combine self-reported SED and SED\% on work and non-work days, estimates were scaled as follows: full-time employment: $(5 / 7)$ x workday estimate $+(2 / 7)$ x non-workday estimate; part-time employment: $(2.5 / 7) \mathrm{x}$ workday estimate $+(4.5 /$ 7) x non-workday estimate; not employed: (7/7) x nonworkday estimate (see Additional file 1: for SB2D questions and scoring). Though not specific to pregnant populations, these questions were included for evaluation as potentially simple instruments to be used in future research.

\section{Statistical analyses}

All analyses were conducted using Stata version 14 (StataCorp, College Station, TX). Demographic and clinical measures were summarized using means and percentages. SED and SED\% from alternative assessment methods (GT3X and self-report questionnaires) were compared to the criterion measure (activPAL) using the Bland-Altman method [38] at each trimester. Pearson's correlations $(r)$, intraclass correlation coefficients (ICC), and reporting of criterion-measured sedentary time distribution across tertiles evaluated the ability of alternative assessment methods to correctly rank women by their participation in sedentary behavior.

\section{Results}

Approximately three fourths of the study population were white and had at least a bachelor's degree (Table 1).
Table 1 Participant Characteristics $(n=58)$

\begin{tabular}{ll}
\hline & mean (SD) or n (\%) \\
\hline Age & $31.5(4.8)$ \\
Race & \\
White & $44(76 \%)$ \\
Black & $7(12 \%)$ \\
Asian & $3(5 \%)$ \\
Multiracial & $4(7 \%)$ \\
Education & \\
$<$ High school & $1(2 \%)$ \\
High school graduate & $15(26 \%)$ \\
College graduate & $14(24 \%)$ \\
Post-graduate degree & $28(48 \%)$ \\
Pre-pregnancy Body Mass Index, kg/m² & $25.1(5.6)$ \\
Gestational Age, week & \\
Visit 1 (1st trimester, 8-13 weeks) & $11.9(1.8)$ \\
Visit 2 (2nd trimester, 20-22 weeks) & $21.2(0.9)$ \\
Visit 3 (3rd trimester, 32-34 weeks) & $33.3(0.9)$ \\
\hline
\end{tabular}

Prior to pregnancy, women had an average BMI of 25.1 (SD 5.6) $\mathrm{kg} / \mathrm{m}^{2}$. Women were assessed, on average, at gestational weeks 11.9 (SD 1.8), 21.2 (SD 0.9), and 33.3 (SD 0.9) (Table 1). By activPAL (the criterion method), women spent approximately $9.5 \mathrm{~h}$ per day and $63 \%$ of their waking time in sedentary behavior in each of the three trimesters (Table 2).

\section{Comparison of Actigraph GT3X to activPAL3 micro}

Average SED was similar between the GT3X and activPAL in all trimesters (Table 2; Fig. 1). Correlations and ICCs of the GT3X with the activPAL ranged from 0.54 to 0.62 ; ICC ranged from 0.52 to 0.61 (Table 2). Bland-Altman plots from each trimester (first row, Fig. 1) revealed minimal systematic bias (underestimation by $<0.5 \mathrm{~h}$ per day), which was nonsignificant in the 1st and 2nd trimester with $p<0.05$ in the 3rd trimester. No discernable pattern of errors across values of SED was observed. However, limits of agreement ranged more than 2 hours in either direction. When women were separated into tertiles based on GT3X SED, criterion SED increased across tertiles in every trimester ( $p$-for- trend $\leq 0.001$; Table 3).

Average SED\% was also similar in each trimester comparing the GT3X to the activPAL (Table 2). Similar to the results for SED, correlations and ICCs for the GT3X as compared to the activPAL for SED\% ranged from 0.64 to 0.66 ; ICC ranged from 0.62 to 0.65 (Table 2). Bland-Altman plots (second row, Fig. 1) again found minimal systematic bias ( $\leq 2 \%$ per day), which was only statistically significant in the first trimester $(p<0.05)$, and no clear pattern of errors. Limits of agreement were approximately $\pm 15 \%$ at each trimester. Criterion SED\% 
Table 2 Sedentary behavior (SED) in hr./day, percent time in SED (SED\%), correlations ( $r$ ), and Intraclass Correlation Coefficients (ICC) between activPAL and GT3X, the Sedentary Behavior Two Domain Questionnaire (SB2D), and the Pregnancy Physical Activity Questionnaire (PPAQ)

\begin{tabular}{|c|c|c|c|c|c|c|c|c|c|}
\hline & \multicolumn{3}{|c|}{ 1st Trimester (<14 weeks) } & \multicolumn{3}{|c|}{ 2nd Trimester (20-22 weeks) } & \multicolumn{3}{|c|}{ 3rd Trimester (32-34 weeks) } \\
\hline & mean (SD) & $r$ & ICC & mean (SD) & $r$ & ICC & mean (SD) & $r$ & ICC \\
\hline \multicolumn{10}{|l|}{ SED, hrs per day } \\
\hline activPAL & $9.63(1.55)$ & 1.0 & 1.0 & $9.54(1.24)$ & 1.0 & 1.0 & $9.43(1.29)$ & 1.0 & 1.0 \\
\hline GT3X $X^{a}$ & $9.50(1.38)$ & 0.62 & 0.61 & $9.08(1.39)$ & 0.58 & 0.55 & $9.08(1.28)$ & 0.54 & 0.52 \\
\hline SB2D & $5.75(2.20)$ & 0.48 & 0.15 & $5.89(2.51)$ & 0.26 & 0.08 & $6.12(3.06)$ & 0.37 & 0.13 \\
\hline$P P A Q^{b}$ & $8.17(3.27)$ & 0.42 & 0.28 & 8.49 (3.39) & 0.24 & 0.14 & $7.95(2.78)$ & 0.28 & 0.18 \\
\hline \multicolumn{10}{|l|}{ SED\% } \\
\hline activPAL & $0.64(0.10)$ & 1.0 & 1.0 & $0.63(0.09)$ & 1.0 & 1.0 & $0.63(0.09)$ & 1.0 & 1.0 \\
\hline GT3X $X^{a}$ & $0.66(0.08)$ & 0.66 & 0.62 & $0.64(0.08)$ & 0.64 & 0.63 & $0.64(0.08)$ & 0.66 & 0.65 \\
\hline SB2D - Likert & $0.57(0.21)$ & 0.44 & 0.32 & $0.55(0.24)$ & 0.52 & 0.31 & $0.60(0.21)$ & 0.45 & 0.33 \\
\hline$P P A Q$ & & $\mathrm{n} / \mathrm{a}$ & & & $\mathrm{n} / \mathrm{a}$ & & & $\mathrm{n} / \mathrm{a}$ & \\
\hline
\end{tabular}

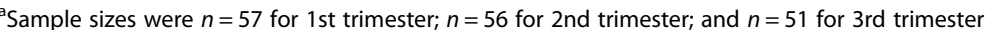

b Sample sizes was $n=57$ for the 2nd trimester

increased across increasing tertiles of GT3X SED\% for each trimester $(p$-for-trend $<0.001$; Table 3 ).

\section{Comparison of self-report to activPAL3 micro}

Average SED reported as hours per day on the SB2D underestimated time spent sedentary by more than $3 \mathrm{~h}$ across trimesters of pregnancy (Table 2; Fig. 2). Correlations ranaged from $r=0.26$ to $r=0.48$ and with ICCs $<$ 0.20 . From the Bland-Altman analyses, SED was significantly underestimated in every trimester $(p<0.001)$, limits of agreement were $\pm 4-5 \mathrm{~h}$, and a pattern emerged in the 2nd the 3rd trimesters where SB2D tended to

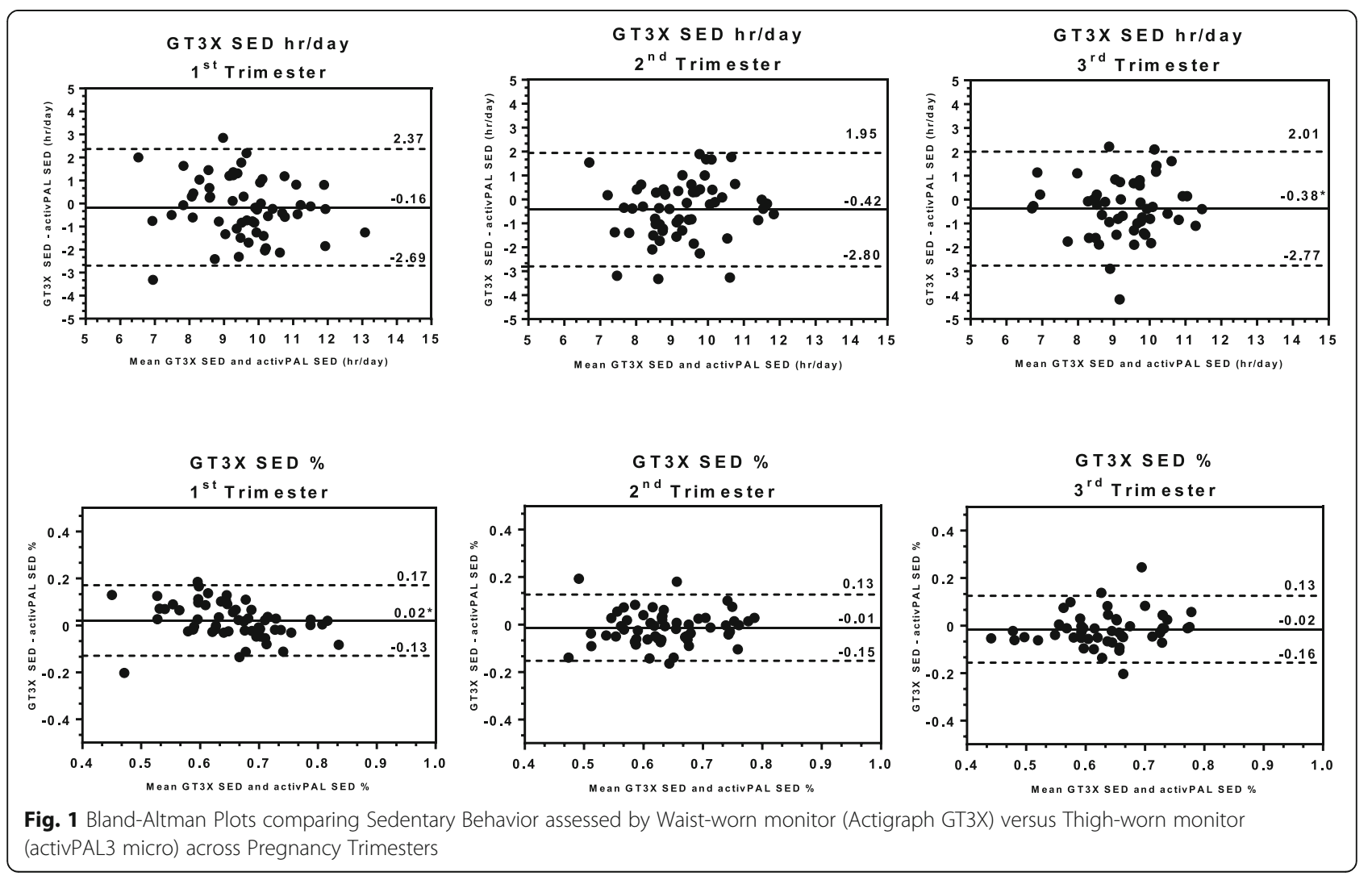


Table 3 Sedentary behavior from the activPAL3 across Tertiles of Sedentary Behavior from the GT3X, the Sedentary Behavior Two Domain Instrument (SB2D), and the Pregnancy Physical Activity Questionnaire (PPAQ)

\begin{tabular}{|c|c|c|c|c|c|c|c|}
\hline & \multicolumn{2}{|c|}{ Tertile 1} & \multicolumn{2}{|c|}{ Tertile 2} & \multicolumn{2}{|c|}{ Tertile 3} & \multirow[t]{2}{*}{$p$-for-linear trend } \\
\hline & mean & (SD) & mean & (SD) & mean & (SD) & \\
\hline \multicolumn{8}{|l|}{ SED hr./day } \\
\hline \multicolumn{8}{|l|}{ GT3Xa } \\
\hline 1st Trimester & 8.58 & (1.34) & 9.84 & (1.09) & 10.54 & (1.55) & $<0.001$ \\
\hline 2nd Trimester & 8.69 & (1.04) & 9.59 & (1.09) & 10.27 & $(1.07)$ & $<0.001$ \\
\hline 3rd Trimester & 8.65 & (1.33) & 9.66 & $(0.97)$ & 10.07 & $(1.05)$ & 0.001 \\
\hline \multicolumn{8}{|l|}{ SB2Q } \\
\hline 1st Trimester & 8.85 & $(1.60)$ & 9.47 & $(1.16)$ & 10.69 & $(1.29)$ & $<0.001$ \\
\hline 2nd Trimester & 9.38 & $(1.20)$ & 9.56 & $(1.25)$ & 9.69 & $(1.31)$ & 0.425 \\
\hline 3rd Trimester & 8.84 & $(0.96)$ & 9.34 & (1.31) & 10.14 & $(1.28)$ & 0.001 \\
\hline \multicolumn{8}{|l|}{$P P A Q^{b}$} \\
\hline 1st Trimester & 8.84 & $(1.45)$ & 9.53 & $(0.99)$ & 10.59 & (1.63) & $<0.001$ \\
\hline 2nd Trimester & 9.07 & $(1.40)$ & 9.63 & $(1.01)$ & 10.00 & (1.14) & 0.018 \\
\hline 3rd Trimester & 8.95 & $(1.12)$ & 9.79 & $(1.02)$ & 9.51 & (1.68) & 0.158 \\
\hline \multicolumn{8}{|l|}{ SED\% } \\
\hline \multicolumn{8}{|l|}{ GT3Xa } \\
\hline 1st Trimester & 0.58 & $(0.09)$ & 0.64 & $(0.09)$ & 0.71 & $(0.08)$ & $<0.001$ \\
\hline 2nd Trimester & 0.58 & $(0.08)$ & 0.62 & $(0.05)$ & 0.70 & $(0.07)$ & $<0.001$ \\
\hline 3rd Trimester & 0.57 & $(0.10)$ & 0.62 & $(0.05)$ & 0.70 & $(0.08)$ & $<0.001$ \\
\hline \multicolumn{8}{|l|}{ SB2D - Likert } \\
\hline 1st Trimester & 0.61 & $(0.09)$ & 0.60 & $(0.08)$ & 0.71 & $(0.09)$ & 0.001 \\
\hline 2nd Trimester & 0.59 & $(0.07)$ & 0.66 & $(0.08)$ & 0.69 & $(0.09)$ & $<0.001$ \\
\hline 3rd Trimester & 0.58 & $(0.07)$ & 0.66 & $(0.08)$ & 0.68 & $(0.12)$ & 0.001 \\
\hline
\end{tabular}

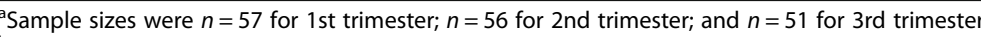

b Sample sizes was $n=57$ for the 2 nd trimester

overestimate SED at low values and underestimate SED at high values (first row, Fig. 2). Tertile analyses (Table 3) revealed that criterion SED increased across tertiles of SB2D SED in the 1st and 3rd trimesters ( $p$-for-trend $\leq$ $0.001)$, but not the 2nd ( $p$-for-trend $=0.425$ ).

Average SED\% from the SB2D (with Likert responses) underestimated SED\% between 2 and 8\%. Correlations with the activPAL ranged from $r=0.44$ to $r=0.52$ across trimesters. Though higher than those for SED, ICCs between SB2D and activPAL for SED\% were still low (ranged from 0.31 to 0.33; Table 2). Bland Altman analyses revealed systematic underestimation in the 1st and 2nd trimesters $(p<0.01)$, but not the 3rd (second row, Fig. 2). A similar pattern of error was observed where the SB2D tended to overestimate SED\% at low values and underestimate SED\% at high values. This resulted in wide limits of agreement that ranged from \pm 36 to $\pm 41 \%$. Criterion SED\% increased across tertiles of SB2D SED\% in each trimester (all $p$-for -trends $<0.001$, Table 3).

Average SED reported as hours per day on the PPAQ significantly underestimated sedentary time across trimesters by approximately $1-1.5 \mathrm{~h}$ (Table 2; Fig. 3). Correlations ranged from 0.24 to 0.42 ; ICC ranged from 0.14 to 0.28 ). Bland-Altman analysis again revealed a similar pattern of errors, with overestimation at low values of SED and underestimation at high values. Limits of agreement were approximately \pm 5.5 to $6.5 \mathrm{~h}$ per day. Criterion SED increased significantly across tertiles of PPAQ SED in the 1st and 2nd (p-for-linear-trends< 0.05 ) but not the 3rd trimesters ( $p$-for-trend $=0.158$ ).

As no total wear time or relative responses were available from the PPAQ, evaluation of the SED\% from the PPAQ was not possible.

\section{Discussion}

With the growing interest of sedentary behavior as a potential risk factor for adverse pregnancy outcomes [11], this study aimed to evaluate the validity of alternative methods versus the criterion activPAL3 micro to measure and rank women by level of sedentary behavior across three trimesters of pregnancy. 


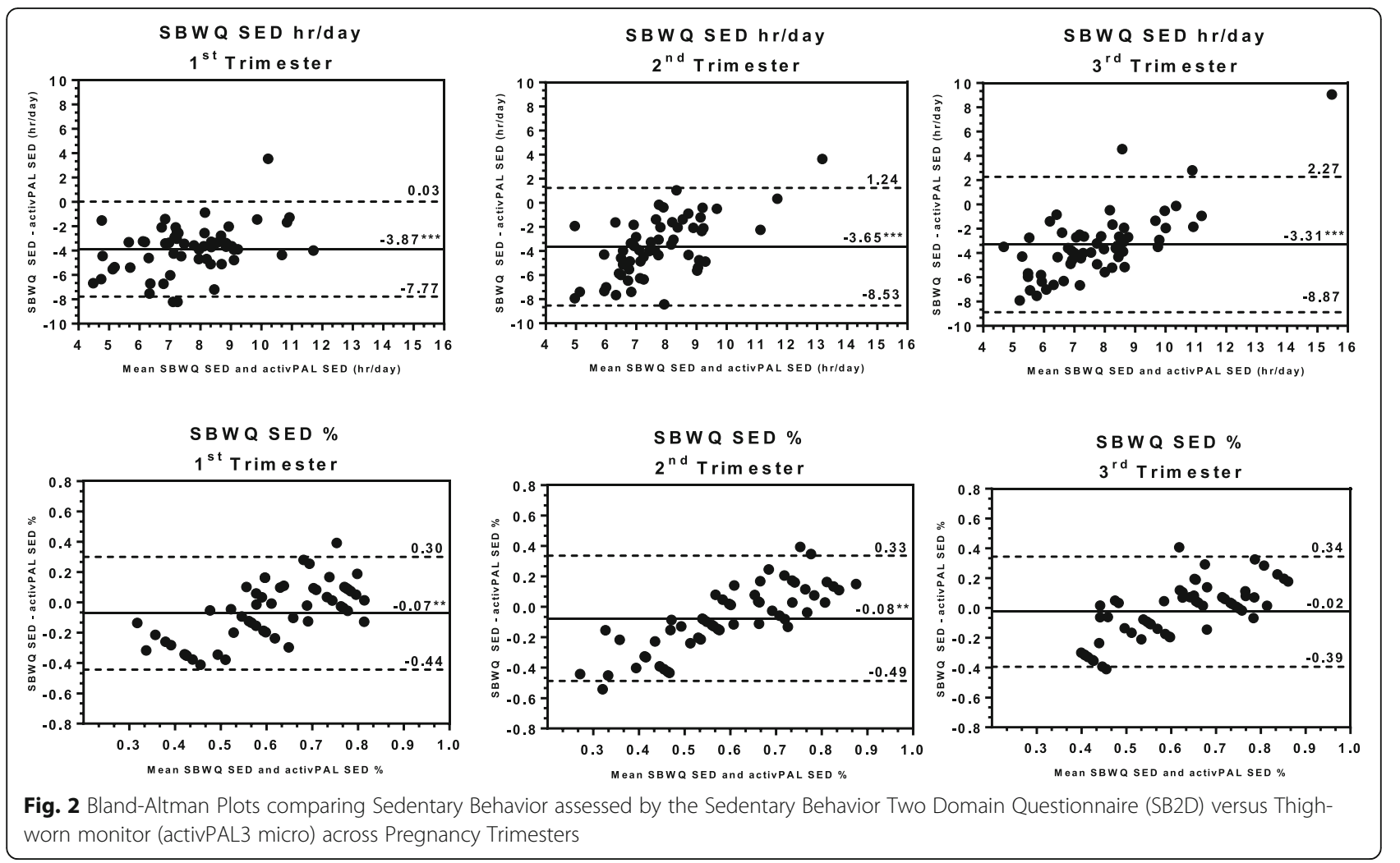

These findings make a contribution as the first study, to our knowledge, to evaluate the validity of sedentary behavior assessed by waist accelerometer versus the current best practice of a thigh-mounted activPAL during pregnancy. Our findings are comparable to similar validation research in non-pregnant adults. A recent validation study in 266 postmenopausal (non-pregnant) women found that waist-worn GT3X assessment resulted in very small differences in average SED $(<0.1 \mathrm{~h}$ per day $)$ but wide limits of agreement (-2.7 to $2.6 \mathrm{~h}$ per day), suggesting unbiased but poor individual-level agreement [15]. This comparison suggests that errors in SED measurement comparing the GT3X to an activPAL are likely similar in pregnant and non-pregnant populations. It is notable that, for SED, the GT3X significantly underestimated SED only in the 2nd and 3rd trimesters and correlations and ICCs decreased across pregnancy. However, this pattern was not observed for SED\%. As such, it is possible that anatomical changes that occur

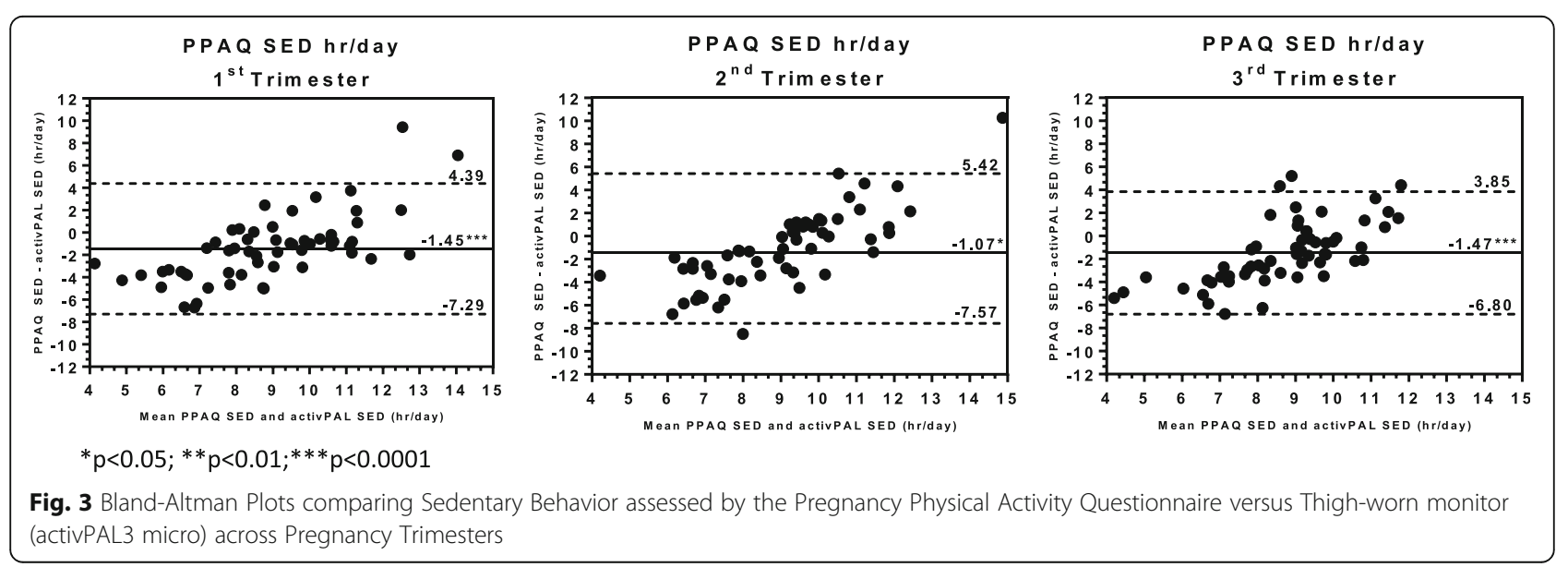


across pregnancy may have increased the error in waist-worn GT3X SED estimated using validated cutpoints in the vertical axis. This possibility is supported by similar research finding that the accuracy of objective monitors for estimating energy expenditure differs across trimesters of pregnancy [21]. Since most of the handful of studies with objective measurement of sedentary behavior in pregnancy have used Actigraph accelerometers [11], our results are helpful when interpreting other study findings in that summary estimates are likely accurate but individuallevel measurement error may attenuate associations with health outcomes [39]. Further, as interventions to decrease sedentary behavior would typically target behaviors in a seated posture such as time spent sitting/reclining watching television, sitting at a desk or table, or lying in bed using a smart phone or tablet, activPAL assessment should be the preferred method when individual precision is important (e.g., to test intervention or longitudinal effects on sedentary behavior). This may be especially important for interventions targeting the second half of pregnancy since imprecision may increase as pregnancy progresses.

We are also unaware of other studies comparing the PPAQ or other self-report measures to activPAL SED in pregnant women. One study used the GT3X as the criterion SED measure and found similar correlations for GPAQ $(r=0.4)$ or PPAQ $(r=0.3)$ across pregnancy [31]. The poor agreement of the PPAQ for measuring SED, especially in later trimesters, is disappointing as this questionnaire is common in pregnancy research studies. Yet, such agreement is comparable to other self-report versus objective measures of moderate-tovigorous physical activity in pregnant women [40]. Further, the PPAQ importantly offers data on domainspecific SED, which can aid in designing interventions [19] and is not measured by the other methodologies evaluated in this analysis. Another important consideration is that we used an updated (DAPA Measurement Toolkit) formula for calculating SED from the PPAQ rather than the originally proposed algorithm. While the updated formula was chosen to correspond most closely with the current consensus definition of sedentary behavior [1], our choice may limit comparisons to previous studies using PPAQ and the originally proposed SED subscale algorithm.

The current study is the first to evaluate the validity of our SB2D questionnaire, asking for separate estimation of non-work and work days and for daily time sitting in hours or on a Likert scale. Assessment of SED duration by the SB2D performed similarly to the PPAQ for ranking of women with $r$ and ICCs all below 0.5 and statistical trends in criterion-measured SED across self-report tertiles in only two of the three trimesters. However, the
SB2D had greater systematic bias as compared to the PPAQ (3-4h vs. $1-1.5 \mathrm{~h}$, respectively). Interestingly, SED\% from our SB2D instrument resulted in the best agreement with activPAL across the self-report instruments we evaluated. At the same time, however, patterned errors and wide limits of agreement were present. Our findings concur with a recent systematic evaluation of self-report instruments of sedentary time compared to activPAL in older adults [36]. Like ours, this study found self-report instruments had poor validity; however, instruments asking participant to report SED\% (via visual analog scale) performed best. Thus, for research where objective monitoring is not feasible, we would recommend our SB2D used herein to measure SED\% (see Additional file 1). Reliability of this instrument and testing of further refinements, such as replacing the Likert Scale with a visual analog scale, are areas for future research.

Strengths of our study include the provision of novel validation data for commonly used and newly developed assessment methods for sedentary behavior, across three trimesters of pregnancy, and in comparison to the activPAL monitor that assesses the most current consensus definition of sedentary behavior. Limitations deserving comment include that the women included in this study were adherent, mostly well-educated participants in a longitudinal cohort study; this may have resulted in improved validity and reduced generalizability of our findings. Further, we only evaluated one commonly used reduction algorithm for the Actigraph GT3X and a few self-reported instruments. Though whether agreement would improve or worsen is not certain, it is possible that different accelerometer data reduction procedures (e.g., different cut-points, non-wear rules, use of vector magnitudes) or self-report questionnaires could have yielded different results. Lastly, concurrent wear of the GT3X and activPAL monitors could tend to increase validity estimates, as compared to the PPAQ which queries SED within the current trimester and the SB2D which had no timeframe.

\section{Conclusions}

The findings herein have implications for interpreting existing sedentary behavior in pregnancy research. First, accelerometer-assessed sedentary behavior likely produces reasonable overall mean estimates, but individual errors (while unbiased) can be substantial. Accelerometer- and activPAL-measured sedentary behavior are not interchangeable. Though still preferable to self-report, using accelerometer-measured sedentary behavior will likely yield attenuated associations and measurement errors could limit the ability to detect intervention effects or changes over time. This may be of particular concern for pregnancy research, as changes across pregnancy 
may be important. The PPAQ sedentary behavior subscale, especially during the later trimesters, should be used with caution. When self-report is the only feasible option, our data suggest that the SB2D querying SED\% on work and non-work days yielded the best estimates of sedentary behavior during pregnancy.

\section{Supplementary information}

Supplementary information accompanies this paper at https://doi.org/10. 1186/s12884-020-2771-z.

Additional file 1. Sedentary Behavior Two Doman (SB2D) Questionniare. SB2D questionnaire and scoring algorithm.

\section{Abbreviations \\ GPAQ: Global Physical Activity Questionnaire; MoM Health: Monitoring Movement and Health; PPAQ: Pregnancy Physical Activity Questionnaire; SB2D: Sedentary Behavior Two Domain Questionnaire; SED: Sedentary} Behavior (hours per day); SED\%: Sedentary Behavior (percent of wear time)

\section{Acknowledgements}

The authors would like to thank the MoM Health participants.

\section{Authors' contributions}

BBG, KMW, and JMC conceived the research idea; BBG, JLP and MAJ collected the data and performed the data analysis; BBG wrote the manuscript; JLP, MAJ, KMW, CPC, and JMC reviewed and provided feedback on the manuscript. All authors read and approved the final manuscript.

\section{Funding}

The MoM Health Study was funded by a Grant-in-Aid from the American Heart Association (17GRNT3340016) with research registry and recruitment support from the University of Pittsburgh Clinical and Translational Science Institute (NIH UL1TR000005). The funding bodies played no role in the design of the study and collection, analysis, and interpretation of data and in writing the manuscript.

\section{Availability of data and materials}

The datasets used and analyzed during the current study are available from the corresponding author on reasonable request (Dr. Bethany Barone Gibbs at bbarone@pitt.edu).

\section{Ethics approval and consent to participate}

All research procedures were approved by the University of Pittsburgh Institutional Review Board. All participants provided written informed consent prior to research participation.

\section{Consent for publication}

N/A

\section{Competing interests}

The authors declare that they have no competing interests.

\section{Author details}

${ }^{1}$ Department of Health and Physical Activity, Clinical and Translational Sciences, University of Pittsburgh, 32 Oak Hill Court, Room 220, Pittsburgh, PA 15261, USA. ${ }^{2}$ Department of Clinical and Translational Sciences, University of Pittsburgh, Pittsburgh, USA. ${ }^{3}$ Department of Health and Human Physiology, Department of Epidemiology, University of lowa, lowa City, USA. ${ }^{4}$ Department of Educational Leadership, Sport Studies, and Educational/ Counseling Psychology, Washington State University, Pullman, USA. ${ }^{5}$ Department of Obstetrics, Gynecology, and Reproductive Sciences, Magee-Womens Hospital of the University of Pittsburgh Medical Center, Pittsburgh, USA.
Received: 22 February 2019 Accepted: 27 January 2020

Published online: 11 February 2020

\section{References}

1. Tremblay MS, Aubert S, Barnes JD, Saunders TJ, Carson V, Latimer-Cheung $A E$, et al. Sedentary behavior research network (SBRN) - terminology consensus project process and outcome. Int J Behav Nutr Phys Act. 2017; 14(1):75.

2. 2018 Physical Activity Guidelines Advisory Committee. 2018 Physical Activity Guidelines Advisory Committee Scientific Report. Washington D.C: U.S Department of Health and Human Services; 2018.

3. Dempsey PC, Larsen RN, Dunstan DW, Owen N, Kingwell BA. Sitting less and moving more: implications for hypertension. Hypertension. 2018:72(5):1037-46.

4. Saunders TJ, Atkinson HF, Burr J, MacEwen B, Skeaff CM, Peddie MC. The acute metabolic and vascular impact of interrupting prolonged sitting: a systematic review and meta-analysis. Sports Med. 2018;48(10):2347-66.

5. Zhai L, Zhang Y, Zhang D. Sedentary behaviour and the risk of depression: a meta-analysis. Br J Sports Med. 2015:49(11):705-9.

6. Sattar N, Greer IA. Pregnancy complications and maternal cardiovascular risk: opportunities for intervention and screening? BMJ. 2002:325(7356):157-60.

7. Nascimento SL, Surita FG, Cecatti JG. Physical exercise during pregnancy: a systematic review. Curr Opin Obstet Gynecol. 2012;24(6):387-94.

8. Hesketh KR, Evenson KR, Stroo M, Clancy SM, Ostbye T, Benjamin-Neelon SE. Physical activity and sedentary behavior during pregnancy and postpartum, measured using hip and wrist-worn accelerometers. Prev Med Rep. 2018;10: 337-45.

9. Borodulin KM, Evenson KR, Wen F, Herring AH, Benson AM. Physical activity patterns during pregnancy. Med Sci Sports Exerc. 2008:40(11):1901-8.

10. Hawkins M, Kim Y, Gabriel KP, Rockette-Wagner BJ, Chasan-Taber L. Sedentary behavior patterns in non-pregnant and pregnant women. Prev Med Rep. 2017;6:97-103.

11. Fazzi C, Saunders DH, Linton K, Norman JE, Reynolds RM. Sedentary behaviours during pregnancy: a systematic review. Int J Behav Nutr Phys Act. 2017; 14(1):32

12. Edwardson CL, Winkler EA, Bodicoat DH, Yates T, Davies MJ, Dunstan DW, et al. Considerations when using the activPAL monitor in field-based research with adult populations. J Sport Health Sci. 2017;6(2):162-78.

13. Chasan-Taber L, Silveira M, Lynch KE, Pekow P, Solomon CG, Markenson G Physical activity and gestational weight gain in Hispanic women. Obesity (Silver Spring, Md). 2014;22(3):909-18.

14. Hawkins M, Chasan-Taber L, Marcus B, Stanek E, Braun B, Ciccolo J, et al. Impact of an exercise intervention on physical activity during pregnancy: the behaviors affecting baby and you study. Am J Public Health. 2014; 104(10):e74-81.

15. Pfister T, Matthews CE, Wang Q, Kopciuk KA, Courneya K, Friedenreich C. Comparison of two accelerometers for measuring physical activity and sedentary behaviour. BMJ Open Sport Exerc Med. 2017;3(1):e000227.

16. Hayes L, Bell R, Robson S, Poston L. Association between physical activity in obese pregnant women and pregnancy outcomes: the UPBEAT pilot study. Ann Nutr Metab. 2014:64(3-4):239-46.

17. Evenson KR, Wen F. Prevalence and correlates of objectively measured physical activity and sedentary behavior among US pregnant women. Prev Med. 2011;53(1-2):39-43.

18. Di Fabio DR, Blomme CK, Smith KM, Welk GJ, Campbell CG. Adherence to physical activity guidelines in mid-pregnancy does not reduce sedentary time: an observational study. Int J Behav Nutr Phys Act. 2015:12:27.

19. Healy GN, Clark BK, Winkler EAH, Gardiner PA, Brown WJ, Matthews CE. Measurement of Adults' sedentary time in population-based studies. Am J Prev Med. 2011;41(2):216-27.

20. Gibbs BB, Hergenroeder AL, Katzmarzyk PT, Lee IM, Jakicic JM. Definition, measurement, and health risks associated with sedentary behavior. Med Sci Sports Exerc. 2015;47(6):1295-300

21. Montoye AH, Dahmen J, Campbell N, Connolly CP. Accuracy of physical activity monitors for steps and calorie measurement during pregnancy walking. J Meas Phys Behav. 2019;2(3):143-56.

22. Koo TK, Li MY. A guideline of selecting and reporting Intraclass correlation coefficients for reliability research. J Chiropr Med. 2016;15(2):155-63.

23. Connolly CP, Coe DP, Kendrick JM, Bassett DR Jr, Thompson DL. Accuracy of physical activity monitors in pregnant women. Med Sci Sports Exerc. 2011; 43(6):1100-5. 
24. Troiano RP, Berrigan D, Dodd KW, Masse LC, Tilert T, McDowell M. Physical activity in the United States measured by accelerometer. Med Sci Sports Exerc. 2008;40(1):181-8.

25. Matthews CE, Chen KY, Freedson PS, Buchowski MS, Beech BM, Pate RR, et al. Amount of time spent in sedentary behaviors in the United States, 2003-2004. Am J Epidemiol. 2008;167(7):875-81.

26. Chasan-Taber L, Schmidt MD, Roberts DE, Hosmer D, Markenson G, Freedson PS. Development and validation of a pregnancy physical activity questionnaire. Med Sci Sports Exerc. 2004;36(10):1750-60.

27. Nascimento SL, Surita FG, Godoy AC, Kasawara KT, Morais SS. Physical activity patterns and factors related to exercise during pregnancy: a cross sectional study. PLoS One. 2015;10(6):e0128953.

28. Chasan-Taber L, Silveira M, Pekow P, Braun B, Manson JE, Solomon CG, et al. Physical activity, sedentary behavior and risk of hypertensive disorders of pregnancy in Hispanic women. Hypertens Pregnancy. 2015;34(1):1-16.

29. Chasan-Taber L, Silveira M, Lynch KE, Pekow P, Braun B, Manson JE, et al. Physical activity before and during pregnancy and risk of abnormal glucose tolerance among Hispanic women. Diabetes Metab. 2014;40(1):67-75.

30. Gibbs BB, Gabriel KP, Reis JP, Jakicic JM, Carnethon MR, Sternfeld B. Crosssectional and longitudinal associations between objectively measured sedentary time and metabolic disease: the coronary artery risk development in young adults (CARDIA) study. Diabetes Care. 2015;38(10):1835-43.

31. Leppe J, Aguilar N, Mena MJ, Besomi M, Roa S. Measuring physical activity and sedentary behaviors in pregnancy: a comparison of accelerometry and questionnaires. Physiotherapy. 2015;101:e861.

32. Cleland CL, Hunter RF, Kee F, Cupples ME, Sallis JF, Tully MA. Validity of the global physical activity questionnaire (GPAQ) in assessing levels and change in moderate-vigorous physical activity and sedentary behaviour. BMC Public Health. 2014;14(1):1255.

33. Gibbs BB, King WC, Davis KK, Rickman AD, Rogers RJ, Wahed A, et al. Objective vs. Self-report Sedentary Behavior in Overweight and Obese Young Adults. J Phys Act Health. 2015; in press.

34. Whitfield GP, Gabriel KK, Kohl HW 3rd. Assessing sitting across contexts: development of the multicontext sitting time questionnaire. Res $Q$ Exerc Sport. 2013;84(3):323-8.

35. Owen N, Sugiyama T, Eakin EE, Gardiner PA, Tremblay MS, Sallis JF. Adults' sedentary behavior determinants and interventions. Am J Prev Med. 2011; 41(2):189-96.

36. Chastin SFM, Dontje ML, Skelton DA, Čukić I, Shaw RJ, Gill JMR, et al. Systematic comparative validation of self-report measures of sedentary time against an objective measure of postural sitting (activPAL). Int J Behav Nutr Phys Act. 2018;15(1):21.

37. Katzmarzyk PT, Church TS, Craig CL, Bouchard C. Sitting time and mortality from all causes, cardiovascular disease, and cancer. Med Sci Sports Exerc. 2009;41(5):998-1005

38. Altman DG, Bland JM. Assessing agreement between methods of measurement. Clin Chem. 2017;63(10):1653-4.

39. Matthews CE, Moore SC, George SM, Sampson J, Bowles HR. Improving selfreports of active and sedentary behaviors in large epidemiologic studies. Exerc Sport Sci Rev. 2012;40(3):118-26.

40. Evenson KR, Chasan-Taber L, Symons Downs D, Pearce EE. Review of selfreported physical activity assessments for pregnancy: summary of the evidence for validity and reliability. Paediatr Perinat Epidemiol. 2012;26(5): 479-94

\section{Publisher's Note}

Springer Nature remains neutral with regard to jurisdictional claims in published maps and institutional affiliations.

Ready to submit your research? Choose BMC and benefit from:
- fast, convenient online submission
- thorough peer review by experienced researchers in your field
- rapid publication on acceptance
- support for research data, including large and complex data types
- gold Open Access which fosters wider collaboration and increased citations
- maximum visibility for your research: over 100M website views per year
At BMC, research is always in progress.
Learn more biomedcentral.com/submissions

